\title{
Camostat mesilate inhibits pro-inflammatory cytokine secretion and improves cell viability by regulating MFGE8 and HMGN1 in lipopolysaccharide-stimulated DF-1 chicken embryo fibroblasts
}

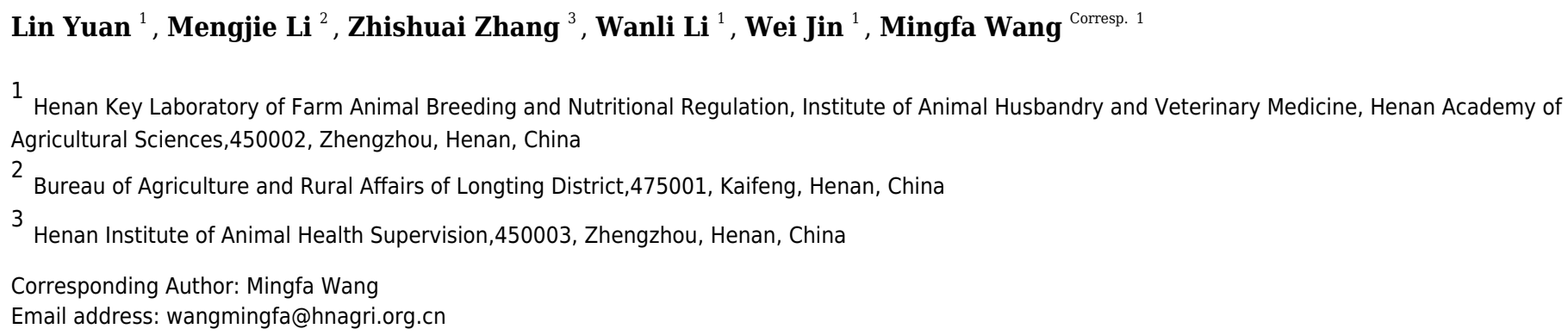

Camostat mesilate (CM) possesses potential anti-viral and anti-inflammatory activities. However, it remains unknown whether CM is involved in lipopolysaccharide (LPS)-mediated inflammatory responses and cell injury. In this project, differentially expressed proteins (DEPs, fold change $\geq \geq 1.2$ or $\leq 0.83$ and $Q$ value $\leq 0.05$ ) in response to LPS stimulation alone or in combination with CM were identified through tandem mass tags (TMT)/mass spectrometry (MS)-based proteomics analysis in DF-1 chicken embryo fibroblasts. The mRNA expression levels of filtered genes were determined by RT-qPCR assay. The results showed that CM alleviated the detrimental effect of LPS on cell viability and inhibited LPSinduced TNF- $\alpha$ and IL- 6 secretions in DF-1 chicken embryo fibroblasts. A total of 141 DEPs that might be involved in mediating functions of both LPS and CM were identified by proteomics analysis in DF-1 chicken embryo fibroblasts. LPS inhibited milk fat globule EGF and factor V/VIII domain containing (MFGE8) expression and induced high mobility group nucleosome binding domain 1 (HMGN1) expression, while these effects were abrogated by CM in DF-1 chicken embryo fibroblasts. MFGE8 knockdown facilitated TNF- $\alpha$ and IL-6 secretions, reduced cell viability, stimulated cell apoptosis in DF-1 chicken embryo fibroblasts co-treated with LPS and CM. HMGN1 loss did not influence TNF- $\alpha$ and IL-6 secretions, cell viability, and cell apoptosis in DF-1 chicken embryo fibroblasts co-treated with LPS and CM. In conclusion, CM exerted anti-inflammatory and pro-survival activities by regulating MFGE8 in LPS-stimulated DF-1 chicken embryo fibroblasts, deepening our understanding of the roles and molecular basis of CM in protecting against Gram-negative bacteria. 
1 Camostat mesilate inhibits pro-inflammatory cytokine secretion and improves cell viability by 2 regulating MFGE8 and HMGN1 in lipopolysaccharide-stimulated DF-1 chicken embryo fibroblasts

3 Lin Yuan ${ }^{1}$, Mengjie Li $^{2}$, Zhishuai Zhang ${ }^{3}$, Wanli Li ${ }^{1}$, Wei Jin ${ }^{1}$, Mingfa Wang ${ }^{1 *}$

4

5 1. Henan Key Laboratory of Farm Animal Breeding and Nutritional Regulation, Institute of Animal Husbandry 6 and Veterinary Medicine, Henan Academy of Agricultural Sciences, Zhengzhou 450002, Henan, China.

7 2. Bureau of Agriculture and Rural Affairs of Longting District, Kaifeng 475001, Henan, China.

8 3. Henan Institute of Animal Health Supervision, Zhengzhou 450003, Henan, China.

9

10 *Correspondence author: Henan Key Laboratory of Farm Animal Breeding and Nutritional Regulation,

11 Institute of Animal Husbandry and Veterinary Medicine, Henan Academy of Agricultural Sciences, No. 116

12 Huayuan Road, Jinshui District, Zhengzhou 450002, P.R. China. Tel: +86-13703937380; E-mail:

13 wangmingfa@hnagri.org.cn. 
15

16

17

\section{Abstract}

Camostat mesilate (CM) possesses potential anti-viral and anti-inflammatory activities. However, it remains unknown whether CM is involved in lipopolysaccharide (LPS)-mediated inflammatory responses and cell injury. In this project, differentially expressed proteins (DEPs, fold change $\geqslant 1.2$ or $\leqslant 0.83$ and $Q$ value $\leqslant 0.05$ ) in response to LPS stimulation alone or in combination with CM were identified through tandem mass tags (TMT)/mass spectrometry (MS)-based proteomics analysis in DF-1 chicken embryo fibroblasts. The mRNA expression levels of filtered genes were determined by RT-qPCR assay. The results showed that CM alleviated the detrimental effect of LPS on cell viability and inhibited LPS-induced TNF- $\alpha$ and IL-6 secretions in DF-1 chicken embryo fibroblasts. A total of 141 DEPs that might be involved in mediating functions of both LPS and CM were identified by proteomics analysis in DF-1 chicken embryo fibroblasts. LPS inhibited milk fat globule EGF and factor V/VIII domain containing (MFGE8) expression and induced high mobility group nucleosome binding domain 1 (HMGN1) expression, while these effects were abrogated by CM in DF-1 chicken embryo fibroblasts. MFGE8 knockdown facilitated TNF- $\alpha$ and IL-6 secretions and reduced cell viability in DF-1 chicken embryo fibroblasts co-treated with LPS and CM. HMGN1 loss did not influence TNF- $\alpha$ and IL-6 secretions, but markedly increased cell viability in DF-1 chicken embryo fibroblasts cotreated with LPS and CM. In conclusion, CM exerted anti-inflammatory and pro-survival activities by regulating MFGE8 and HMGN1 in LPS-stimulated DF-1 chicken embryo fibroblasts, deepening our understanding of the roles and molecular basis of CM in protecting against Gram-negative bacteria.

Key words: Camostat mesilate, lipopolysaccharide, fibroblast, chicken, inflammation, viability, MFGE8, HMGN1

\section{Introduction}

Camostat mesilate (CM, Foipan $\left.{ }^{\mathrm{TM}}\right)$, a protease inhibitor, has been used for the treatment of multiple diseases such as chronic pancreatitis and recessive dystrophic epidermolysis bullosa in human [1-3]. Moreover, some studies have proposed that CM (an inhibitor of TMPRSS2 serine protease) might act as a candidate drug against SARS-CoV-2 and COVID-19 given its inhibitory effect on host cell entry of SARS-CoV-2 and its protective effects against SARS-CoV-induced mouse death $[1,4,5]$. In addition, CM has potential anti-viral 
42 activities against influenza and parainfluenza viruses [6, 7]. Furthermore, previous in vitro and in vivo studies

43 have demonstrated that CM exerts anti-inflammatory activity in LPS-activated rat monocytes and chronic

44 pancreatitis and genetically diabetic rat models [8, 9]. For instance, Jia et al. have pointed out that CM can

45 inhibit the expression of interleukin (IL)-1 $1 \beta$, tumor necrosis factor- $\alpha$ (TNF- $\alpha$ ), and IL-6 in the fibrotic or degenerative regions of the pancreas in obese and diabetic rats [9]. However, few studies have been performed currently to investigate the association of CM and Gram-negative bacteria-mediated inflammatory responses and cell injury.

Gram-negative bacteria, a group of pathogens infecting all eukaryotes, can trigger multitudinous diseases (e.g. infertility and fowl cholera) in birds including chickens [10-12]. Moreover, birds carrying bacteria can transmit pathogens to other animals and human, which seriously influences public health and food safety [13-15]. Toxins and virulent factors generated by bacteria play vital roles in the determination of the pathogenesis of these pathogens [14]. Lipopolysaccharide (LPS), also known as endotoxin, is one of the main virulent factors of most Gram-negative bacteria [12]. It has reported that toll-like receptor (TLR) 4 signaling pathway serves as a crucial player in the response to extracellular LPS or bacteria $[16,17]$. For instance, the expression levels of TLR4, TLR4 pathway-related genes (e.g. myeloid differentiation primary response 88, TNF receptor associated factor 6, interferon regulatory factor 7) and inflammatory cytokines (e.g. IL-2, IL-6, IL-1 $\beta$, and TNF- $\alpha$ ) have been found to be dysregulated in peripheral mononuclear blood cells (PBMCs) of Aseel, Ghagus, Dahlem red, and Broiler chickens upon LPS treatment [18]. Also, the allelic variation in TLR4 has been reported to be associated with the resistance to Salmonella enterica serovar Typhimurium infection in chickens [19]. Fibroblasts, a kind of antigen-presenting cell, can respond to the signals related to injuries and pathogens (e.g. bacteria, viruses) by TLRs including TLR4 [20,21]. For example, Zhao et al. have demonstrated that the expression levels of TLR4 and TLR4 downstream genes (IL-6, MHC II, and IL-1 $\beta$ ) are dysregulated in duck embryo fibroblasts following LPS stimulation [22]. Moreover, not only live bacteria but also LPS can induce pro-inflammatory responses and subsequent cell injury in fibroblasts [23, 24]. Fibroblasts, one member of the connective-tissue cell family, function as vital players in the development of multiple diseases such as organ damage/fibrosis [20, 25] and arthritis [26]. Moreover, fibroblasts have been identified as the sentinel cells 
69 keratitis [27] and periodontitis [28, 29]. Additionally, fibroblasts are involved in the initiation, modulation, and

70 maintenance of inflammation [30].

71 In this text, the effects and molecular mechanisms of $\mathrm{CM}$ on Gram-negative bacteria-induced inflammation

72 and cell injury were preliminarily explored by ELISA assay, CCK-8 assay and tandem mass tags (TMT)/mass

73 spectrometry-based proteomics analysis in LPS-treated DF-1 chicken embryo fibroblasts. Moreover, we

74 further investigated whether high-mobility group (HMG) nucleosome binding domain 1 (HMGN1) and milk

75 fat globule epidermal growth factor 8 (MFGE8) were involved in the regulation of CM functions in LPS-

76 treated DF-1 chicken embryo fibroblasts.

Materials and methods

79 Cell culture

80 DF-1 chicken embryo fibroblasts (CRL-12203, American Type Culture Collection, Manassas, VA, USA) were

81 cultured in Dulbecco's Modified Eagle Medium (Thermo Scientific, Waltham, MA, USA) containing 10\%

82 fetal bovine serum (Thermo Scientific) at $39^{\circ} \mathrm{C}$, and treated with different concentrations of LPS (Sigma-

83 Aldrich, St. Louis, MO, USA) for $6 \mathrm{~h}$.

\section{Reagents}

85 Lipopolysaccharides (from Escherichia coli) (L2630) were purchased from Sigma-Aldrich. CM was obtained 86 from ChemeGen Lnc. (Los Angeles, California, USA). Small interference RNAs (siRNAs) targeting MFGE8

87 (si-MFGE8\#1 and si-MFGE8\#2), HMGN1 (si-HMGN1\#1 and si-HMGN1\#2), and a scrambled siRNA control

88 (si-NC) were synthesized by GenePharma Co., Ltd. (Shanghai, China). Cells were transfected with corresponding siRNAs using Lipofectamine 2000 reagent (Thermo Scientific) according to the instructions of the manufacturer. SiRNA sense sequences were presented in Supplementary Table 1.

\section{$91 \quad$ RT-qPCR assay}

92 Total RNA was extracted from DF-1 chicken embryo fibroblasts using Trizol reagent (Thermo Scientific).

93 Next, RNA was reversely transcribed into cDNA first strand using M-MLV Reverse Transcriptase (Thermo 94 Scientific) following the manufacturer's instructions. The mRNA expression levels of MFGE8, myristoylated 95 alanine-rich protein kinase $\mathrm{C}$ substrate (MARCKS), HMGN1, high mobility group nucleosomal binding 
96

97

domain 4 (HMGN2), and GAPDH were measured through the real-time quantitative system using cDNA template, SYBR Green PCR Master Mix (Thermo Scientific) and corresponding quantitative primers. The primer sequences were presented as below: 5'-CGATCTGAACTACATGGTTTAC-3' (forward) and 5'TTCACTCTGATGCGGTCCAC-3' (reverse) for GAPDH, 5'-GCCACGTCAAAGACTGGAAAC-3' (forward) and 5'-AATGCCTGCACCCCCTTATAC-3' (reverse) for HMGN1, 5'TGTTAGCACACAGACCGCTT-3'(forward) and 5'-TTCACTCTGATGCGGTCCAC-3' (reverse) for HMGN2， 5'-TTACCACCATTCCAACGGGC-3' (forward) and 5'-GATCCCTTATCGACCCACCC-3' (reverse) for MARCKS, 5'-CAAGGTTTTCCAGGGCAACG-3' (forward) and 5'GCAACCTGCCGTGTTGAAAT-3' (reverse) for MFGE8.

\section{ELISA assay}

DF-1 chicken embryo fibroblasts transfected with or without si-NC, si-MFGE8, or si-HMGN1 were treated with LPS for $6 \mathrm{~h}$ and then incubated with CM for an additional $24 \mathrm{~h}$. TNF- $\alpha$ and IL-6 secretion levels were measured using corresponding ELISA kits (MEIMIAN Biotechnology Co., Ltd., Wuhan, China) following the manufacturer's protocols.

\section{CCK-8 assay}

Cell viability was determined through Cell Counting Kit-8 (Beyotime Biotechnology, Shanghai, China) according to the manufacturer's protocols. Briefly, cells were inoculated into 96-well plates. At the indicated time points after treatment, $10 \mu \mathrm{l}$ of CCK-8 solution was added into each well (100 $\mu 1$ medium/well). Three hours later, the optical density (OD) values were determined at $450 \mathrm{~nm}$.

\section{Cell apoptosis detection}

Cell apoptotic rate was determined by Annexin V-FITC Apoptosis Staining/Detection Kit (Solarbio, Beijing China). Briefly, DF-1 cells were collected and re-suspended in $500 \mu 1$ of 1 x Binding Buffer. Then, cells were co-incubated with $5 \mu \mathrm{l}$ of Annexin V-FITC and $5 \mu \mathrm{l}$ of PI at room temperature in a dark room. Cell apoptotic patterns were analyzed by flow cytometry (Becton Dickinson Co. CA, USA).

\section{TMT-based proteomics analysis}

Cell samples (3 biological replicates) were lysed using the protein lysis buffer, sonicated, and centrifuged to obtain cell lysates containing proteins. After treated with dithiothreitol (DTT) and iodoacetamide (IAM), 
123 proteins were digested into peptide segments with trypsin. Next, the peptide segments post enzymolysis were 124 labeled with TMT and fractionated on an L-3000 high-performance liquid chromatography (HPLC) system. 125 Finally, fractions were analyzed and detected using EASY-nLCTM 1200 Ultra High-Performance Liquid 126 Chromatography (UHPLC, Thermo Scientific) and Q Exactive HF-X Mass Spectrometer (Thermo Scientific). 127 Proteins were identified using Proteome Discoverer 2.2 (Thermo Scientific). Proteins were quantified using

Maxquant software. GO and KEGG pathway enrichment analyses were performed using the KOBAS 3.0 website (http://kobas.cbi.pku.edu.cn/kobas3).

\section{Statistical analysis}

In the proteomics analysis, differences between groups were analyzed using Student's $t$-test. $P$ values obtained from Student's t-test were corrected through false discovery rate (FDR). The $P$ values after FDR correction were termed as Q values. Proteins were regarded as differentially expressed at the fold change $\geqslant 1.2$ or $\leqslant 0.83$ and $\mathrm{Q}$ value $\leqslant 0.05$.

In the cell experiments, data analysis was conducted using GraphPad Prism software 7.0 (La Jolla, CA, USA) with the results presenting as mean \pm standard deviation. Differences between groups were compared using Student's $t$-test. Differences among groups were analyzed through one-way ANOVA (Tukey's post-hoc test) or two-way ANOVA (Sidak post hoc test). Differences were regarded as statistically significant when the $P$ value was less than 0.05 .

\section{Results}

CM weakened LPS-induced pro-inflammatory cytokine secretion and cell injury in DF-1 chicken embryo fibroblasts.

Firstly, our data revealed that LPS facilitated TNF- $\alpha$ and IL-6 secretions and decreased cell viability in a concentration-dependent manner with the strongest effect at the concentration of $10 \mu \mathrm{g} / \mathrm{ml}$ (Fig. 1A-1C). Hence, $10 \mu \mathrm{g} / \mathrm{ml}$ of LPS was used to construct the cell model. Moreover, our study demonstrated that CM dose-dependently inhibited the secretions of TNF- $\alpha$ and IL-6 in the LPS (10 $\mu \mathrm{g} / \mathrm{ml})$-exposed DF-1 cell model (Fig. 1D and 1E). Additionally, CM increased cell viability in a time- and concentration-dependent manner in LPS-treated DF-1 chicken embryo fibroblasts (Fig. 1F). These data suggested that CM could protect DF-1 
150

151

152

153

154

155

156

157

158

159

160

161

162

163

164

165

166

167

168

169

170

171

172

173

174

175

176

chicken embryo fibroblasts from LPS-induced inflammation and cell injury.

Identification of dysregulated proteins in response to LPS stimulation alone or in combination with CM in DF-1 chicken embryo fibroblasts.

Next, dysregulated proteins in DF-1 chicken embryo fibroblasts upon the treatment of LPS alone or in combination with CM were screened out by TMT/mass spectrometry (MS)-based proteomics analysis. Results showed that 273 proteins were differentially expressed (182 up-regulated, 91 down-regulated) in LPS treated DF-1 cell group (DL group) compared to the untreated cell group (D group) (Fig. 2A and 2B). Moreover, 102 proteins were highly expressed and 547 proteins were low expressed in DF-1 chicken embryo fibroblasts cotreated with LPS and CM (DLE group) than that in DF-1 chicken embryo fibroblasts treated with LPS alone (DL group) (Fig. 2A and 2B). In addition, 26 common proteins were identified by Venn analysis among downregulated proteins in DL vs D group and up-regulated proteins in DLE vs DL group (Fig. 2A). Also, 115 proteins were found to be up-regulated in DL vs D group and down-regulated in DLE vs DL group (Fig. 2B). These proteins with the converse change trends in DLE vs DL and DL vs D groups might be involved in the responses of DF-1 chicken embryo fibroblasts to LPS and CM. The heat map of the proteins with converse change trends in DLE vs DL and DL vs D groups was presented in Supplementary Fig. 1 and corresponding protein information was displayed in Supplementary Table 2. Next, GO and KEGG enrichment analysis for these proteins with the converse change trends in DLE vs DL and DL vs D groups were carried out to decrypt the protective mechanisms of CM in LPS-stimulated DF-1 chicken embryo fibroblasts. The top 20 GO enrichment terms based on biological processes, cellular components, and molecular functions were presented in Fig. 2C-2E. GO enrichment analysis showed that these proteins were mainly involved in the regulation of biological processes such as cellular/metabolic/biological processes, cellular component organization or biogenesis, gene expression, protein/nitrogen compound/peptide/amide transport, and apoptotic cell clearness (Fig. 2C, Supplementary Table 3). KEGG enrichment analysis revealed that 7 KEGG pathways (ribosome, steroid biosynthesis, RNA transport, MAPK signaling pathway, spliceosome, herpes simplex virus 1 infection, and salmonella infection) were significantly enriched by these proteins (Fig. 2F, Supplementary Table 4). These enrichment analyses suggested that CM could trigger the notable dysregulation of a host of essential genes related to cell development.

PeerJ reviewing PDF | (2021:01:57494:1:1:NEW 2 Jul 2021) 
177

178

179

180

181

182

183

184

185

186

187

Effects of LPS alone or in combination with CM on the expression of MFGE8, MARCKS, HMGN1, and HMGN2 in DF-1 chicken embryo fibroblasts.

Among proteins with the inverse alteration trends in DLE vs DL and DL vs D groups, 4 proteins (MFGE8 [31], MARCKS [32], HMGN1 [33], and HMGN2 [34]) related to inflammation were screened out for further explorations according to previous documents. Annotation analysis revealed that MFGE8 was involved in the regulation of multiple pathways or biological processes such as protein metabolism, post-translation protein modification, insulin-like growth factor (IGF) transport and uptake by insulin-like growth factor binding proteins (IGFBPs), phagocytosis, and apoptotic cell clearance (Supplementary Table 5). MARCKS participated in actin/protein kinase C/calmodulin binding, actin filament organization, central nervous system development, and actin crosslink formation (Supplementary Table 5). HMGN1 could regulate nucleotideexcision repair, chromatin organization, transcription by RNA polymerase II, UV-B/UV-C response, and NAD + ADP-ribosyltransferase activity (Supplementary Table 5). HMGN2 was implicated in chromatin organization (Supplementary Table 5). TMT-MS data revealed that MFGE8 expression was notably increased in DLE vs DL group (1.66-fold) and markedly reduced in DL vs D group (0.81-fold) (Supplementary Table 2). The fold-change values of MARCKS, HMGN1, and HMGN2 in the DLE vs DL group are 0.61, 0.59, and 0.55, respectively. The fold-change values of MARCKS, HMGN1, and HMGN2 in the DL vs D group are 1.39, 1.54, 1.89, respectively (Supplementary Table 2). Consistent with the TMT-MS outcomes, RT-qPCR assay also disclosed that MFGE8 expression was markedly reduced, and HMGN1 and HMGN2 expression was notably increased in LPS-stimulated DF-1 chicken embryo fibroblasts (DL group) versus un-stimulated cells (D group) (Fig. 3A, 3C, 3D). However, there was no obvious alteration in MARCKS expression in LPSstimulated DF-1 chicken embryo fibroblasts compared to untreated cells (Fig. 4B). In line with TMT-MS data, higher MFGE8 expression and lower HMGN1 expression were also validated by RT-qPCR assay in DF-1 chicken embryo fibroblasts with the combined treatment of LPS and CM (DLE group) compared to cells treated with LPS alone (Fig. 3A, 3C). Nevertheless, the addition of CM did not influence MARCKS and HMGN2 expression in LPS-treated DF-1 chicken embryo fibroblasts (Fig. 3B, 3D). Given the consistency of RT-qPCR and proteomics data, HMGN1 and MFGE8 were selected for further investigations.

CM exerted its functions through regulating MFGE8 and HMGN1 expression in LPS-treated DF-1 
204

205

206

207

208

209

210

211

212

213

214

215

216

217

218

219

220

221

222

223

224

225

226

227

228

229

230

chicken embryo fibroblasts.

To further investigate the functions of MFGE8 and HMGN1, si-NC and siRNAs targeting MFGE8 and HMGN1 were synthesized. Knockdown efficiency analyses revealed that the introduction of si-MFGE8\#1 led to the notable down-regulation of MFGE8 mRNA level in DF-1 chicken embryo fibroblasts compared with the si-NC group, while si-MFGE8\#2 could not effectively silence MFGE8 in DF-1 chicken embryo fibroblasts (Fig. 4A). Also, a noticeable reduction of HMGN1 mRNA expression level was observed in DF-1 chicken embryo fibroblasts transfected with si-HMGN1\#1 or si-HMGN1\#2 than that in cells transfected with si-NC (Fig. 4B). Considering the stronger knockdown outcomes of si-MFGE8\#1 and si-HMGN1\#2 on their respective targets, si-MFGE8\#1 and si-HMGN1\#2 were selected in the subsequent loss-of-function explorations. Functional analyses revealed that MFGE8 knockdown could weaken the effects of CM on TNF- $\alpha$ and IL-6 secretions, cell viability, and cell apoptosis in LPS-treated DF-1 chicken embryo fibroblasts (Fig. 4C4F). Moreover, our TMT-MS outcomes showed that CM treatment led to the notable increase of MFGE8 in LPS-treated DF-1 chicken embryo fibroblasts. These data suggested that CM could inhibit TNF- $\alpha$ and IL-6 secretions and improved cell viability by up-regulating MFGE8 in LPS-treated DF-1 chicken embryo fibroblasts. Additionally, HMGN1 knockdown did not have statistically significant influence on TNF- $\alpha$ and IL-6 secretions, cell viability and apoptosis in DF-1 chicken embryo fibroblasts co-treated with LPS and CM (Fig. 4C-4F).

\section{Discussion}

In this text, our data revealed that LPS stimulated the secretions of pro-inflammatory factors (TNF- $\alpha$ and IL-6) and reduced cell viability in DF-1 chicken embryo fibroblasts, suggesting the successful establishment of LPSinduced inflammation and cell injury model. Similarly, fibroblasts have been found to sense LPS and LPS stimulation led to the notable increase in the expressions of pro-inflammatory cytokines (e.g. IL-1 $\beta$, TNF- $\alpha$, IL-8, and IL-6) in human gingival fibroblasts [35]. LPS induced cell apoptosis and inhibited cell proliferation and migration in Guinea pig fibroblasts [36].

Considering the anti-inflammatory activity of $\mathrm{CM}$, the effects of $\mathrm{CM}$ on pro-inflammatory factor secretion and cell viability along with its molecular mechanisms were further investigated in LPS-stimulated DF-1 chicken 
231

232

233

234

235

236

237

238

239

240

241

242

243

244

245

246

247

248

249

250

251

252

253

254

255

256

257

embryo fibroblasts. Our results showed that CM inhibited the secretions of TNF- $\alpha$ and IL-6 and increased cell viability in LPS-stimulated DF-1 chicken embryo fibroblasts, suggesting that CM could protect chicken embryo fibroblasts from LPS-induced inflammation and cell damage.

Next, proteins that might be involved in mediating the anti-inflammatory and pro-survival activities of $\mathrm{CM}$ were screened out by TMT/MS-based proteomics analysis in the LPS-induced DF-1 cell models. Among proteins with the converse change trends in DLE vs DL and DL vs D groups, HMGN1 and MFGE8 were selected out to further investigate in virtue of the consistency of proteomics data and RT-qPCR outcomes.

HMGN1, formerly named as HMGN14, has been found to be implicated in multiple pathophysiologic processes such as inflammation, immunity, and fibrosis [33, 37, 38]. For instance, Yang et al. demonstrated that HMGN1 stimulated pro-inflammatory cytokine (e.g. IL-6, IL-8, TNF) production, facilitated dendritic cell maturation, induced leukocyte recruitment, and enhanced immune responses induced by ovalbumin or/and LPS in mice [33]. Recombinant HMGN1 induced the production of pro-inflammatory cytokines such as IL-6, TNF- $\alpha$, and IL-1 in human peripheral blood mononuclear cells [39]. Given the close link between HMGN1 and inflammation, we further investigated whether $\mathrm{CM}$ could exert its functions by regulating HMGN1 expression in LPS-treated DF-1 chicken embryo fibroblasts. Our data revealed that HMGN1 knockdown or not did not influence TNF- $\alpha$ and IL-6 secretions, cell viability and apoptosis in DF-1 chicken embryo fibroblasts co-treated with LPS and CM.

Milk fat globule epidermal growth factor 8 (MFGE8), a peripheral membrane glycoprotein, also has been found to be involved in inflammation and immunity [31,40]. For instance, Nakaya et al. demonstrated that MFGE8 knockout enhanced apoptotic cell accumulation and inflammatory responses, and cardiac dysfunction in myocardial infarction mice [41]. MFGE8 weakened LPS-induced pro-inflammatory responses in mouse macrophages, microglia, and brains [42, 43]. Our proteomics and RT-qPCR data showed that MFGE8 expression was notably reduced in LPS-treated DF-1 chicken embryo fibroblasts compared to untreated cells, but was markedly increased in DF-1 chicken embryo fibroblasts co-treated with LPS and CM compared to cells treated with LPS alone. Functional analyses revealed that MFGE8 knockdown weakened CM-mediated pro-survival and anti-inflammatory activities in LPS-treated DF-1 chicken embryo fibroblasts. Because CM is an inhibitor of TMPRSS2, the potential link between TMPRSS2 and MFGE8 was established using the 
258 STITCH website (http://stitch.embl.de/cgi/). Results showed that the possible association between TMPRSS2

259 and MFGE8 might be mediated by calcium ions (Supplementary Fig. 2).

260 However, protein antibodies specific for chicken are relatively poor. The potential targets of CM identified by

261 proteomics analysis and other experimental approaches need to be further validated. To examine the effect of

$262 \mathrm{CM}$ on protein expression of CM targets in chicken cells, we need to align the protein sequences of CM targets

263 between chicken and human to examine whether protein antibody specific for human can be used in chicken. If

264 the percentage of identical sequences reaches more than $80 \%$, we would try to measure the protein levels of 265 these targets in chicken using the antibody specific for human. In our project, the identity between human and 266 chicken MFGE8 protein sequences was only about 50\%. So, we did not detect the protein level of chicken

267 MFGE8 using the antibody specific for human MFGE8 protein. Because the identity of human and chicken

268 MFGE8 protein sequences is relatively low, the alterations that occurred in human cell lines cannot represent

269 the effects in chickens.

270 Taken together, our data revealed that CM alleviated the detrimental effect of LPS on cell viability and

271 weakened LPS-induced pro-inflammatory responses partly by regulating MFGE8 and HMGN1 expression in

272 chicken embryo fibroblasts. These data suggested that CM might help chicken embryo fibroblasts fight against

273 Gram-negative bacteria infection by regulating MFGE8, deepening our understanding of the roles and protective mechanisms of CM in Gram-negative bacteria-induced inflammatory responses and cell injury and hinting at the potential value of CM in protecting cells from Gram-negative bacteria. Moreover, a multitude of proteins that might be involved in regulating DF-1 cell responses to Gram-negative bacterium infection alone or in combination with CM treatment were screened out by TMT-based proteomics analysis.

Data Availability Statement

280 The data and material presented in this manuscript is available from the corresponding author on reasonable 281 request.

\section{Conflict of interest}

283 The authors declare none conflict of interest.

\section{$284 \quad$ Funding}


285 This research was supported by Science-Technology Foundation for Outstanding Young Scientists of Henan

286 Academy of Agricultural Sciences (Grant no. 2020YQ20).

287 Authors' contributions

288 LY designed and performed most of the experiments, and wrote the manuscript. ML, ZZ contributed to data analysis and involved in the study design. WL and WJ contributed to the experimental work. MW made contributions to the study design, data analysis and manuscript revision. All authors have read and approved 291 the final version to be published.

\section{References}

294 1. McKee DL, Sternberg A, Stange U, Laufer S, Naujokat C: Candidate drugs against SARS-CoV-2 and COVID-19. Pharmacol Res 2020, 157:104859.

2. Ramsey ML, Nuttall J, Hart PA: A phase 1/2 trial to evaluate the pharmacokinetics, safety, and efficacy of NI-03 in patients with chronic pancreatitis: study protocol for a randomized controlled trial on the assessment of camostat treatment in chronic pancreatitis (TACTIC). Trials 2019, 20(1):501.

3. Ikeda S, Manabe M, Muramatsu T, Takamori K, Ogawa H: Protease inhibitor therapy for recessive dystrophic epidermolysis bullosa. In vitro effect and clinical trial with camostat mesylate. Journal of the American Academy of Dermatology 1988, 18(6):1246-1252.

4. Uno Y: Camostat mesilate therapy for COVID-19. Internal and emergency medicine 2020:1-2.

5. Hoffmann M, Kleine-Weber H, Schroeder S, Krüger N, Herrler T, Erichsen S, Schiergens TS, Herrler G, Wu NH, Nitsche A et al: SARS-CoV-2 Cell Entry Depends on ACE2 and TMPRSS2 and Is Blocked by a Clinically Proven Protease Inhibitor. Cell 2020, 181(2):271-280.e278.

6. Yamaya M, Shimotai Y, Hatachi Y, Lusamba Kalonji N, Tando Y, Kitajima Y, Matsuo K, Kubo H, Nagatomi R, Hongo $\mathrm{S}$ et al: The serine protease inhibitor camostat inhibits influenza virus replication and cytokine production in primary cultures of human tracheal epithelial cells. Pulmonary pharmacology \& therapeutics 2015, 33:66-74.

7osoya M, Matsuyama S, Baba M, Suzuki H, Shigeta S: Effects of protease inhibitors on 
312

3138

replication of various myxoviruses. Antimicrobial agents and chemotherapy 1992, 36(7):1432-1436.

8. Gibo J, Ito T, Kawabe K, Hisano T, Inoue M, Fujimori N, Oono T, Arita Y, Nawata H. Camostat mesilate attenuates pancreatic fibrosis via inhibition of monocytes and pancreatic stellate cells activity. Lab Invest 2005, 85(1):75-89.

9. Jia D, Taguchi M, Otsuki M: Synthetic protease inhibitor camostat prevents and reverses dyslipidemia, insulin secretory defects, and histological abnormalities of the pancreas in genetically obese and diabetic rats. Metabolism: clinical and experimental 2005, 54(5):619-627.

10. Deb K, Chatturvedi MM, Jaiswal YK: Gram-negative bacterial endotoxin- induced infertility: a birds eye view. Gynecologic and obstetric investigation 2004, 57(4):224-232.

11. Harper M, Boyce JD: The Myriad Properties of Pasteurella multocida Lipopolysaccharide. Toxins 2017, 9(8).

12. Neyen C, Lemaitre B: Sensing Gram-negative bacteria: a phylogenetic perspective. Current opinion in immunology 2016, 38:8-17.

13. Gargiulo A, Fioretti A, Russo TP, Varriale L, Rampa L, Paone S, De Luca Bossa LM, Raia P, Dipineto L: Occurrence of enteropathogenic bacteria in birds of prey in Italy. Letters in applied microbiology 2018, 66(3):202-206.

14. Abebe E, Gugsa G, Ahmed M: Review on Major Food-Borne Zoonotic Bacterial Pathogens. Journal of tropical medicine 2020, 2020:4674235.

15. Nga VT, Ngoc TU, Minh LB, Ngoc VTN, Pham VH, Nghia LL, Son NLH, Van Pham TH, Bac ND, Tien TV et al: Zoonotic diseases from birds to humans in Vietnam: possible diseases and their associated risk factors. 2019, 38(6):1047-1058.

16. Mazgaeen L, Gurung P: Recent Advances in Lipopolysaccharide Recognition Systems. International journal of molecular sciences 2020, 21(2).

17. Brownlie R, Allan B. Avian toll-like receptors. Cell Tissue Res 2011, 343(1):121-30.

18. Karnati HK, Pasupuleti SR, Kandi R, Undi RB, Sahu I, Kannaki TR, Subbiah M, Gutti RK. TLR-4 signalling pathway: MyD88 independent pathway up-regulation in chicken breeds upon LPS treatment. Vet Res Commun 2015, 39(1):73-8. 
339 19. Leveque G, Forgetta V, Morroll S, Smith AL, Bumstead N, Barrow P, Loredo-Osti JC, Morgan K,

340

341

34220.

343

34421.

345

346

34722.

348

349

350

351

352

353

354

355

356

357

358

359

360

361

362

363
Malo D. Allelic variation in TLR4 is linked to susceptibility to Salmonella enterica serovar Typhimurium infection in chickens. Infect Immun 2003, 71(3):1116-24.

Turner JD, Naylor AJ, Buckley C, Filer A, Tak PP: Fibroblasts and Osteoblasts in Inflammation and Bone Damage. Advances in experimental medicine and biology 2018, 1060:37-54.

Pinheiro CR, Coelho AL, de Oliveira CE, Gasparoto TH, Garlet GP, Silva JS, Santos CF, Cavassani KA, Hogaboam CM, Campanelli AP: Recognition of Candida albicans by gingival fibroblasts: The role of TLR2, TLR4/CD14, and MyD88. Cytokine 2018, 106:67-75.

22. Zhao W, Huang Z, Chen Y, Zhang Y, Rong G, Mu C, Xu Q, Chen G. Molecular cloning and functional analysis of the duck TLR4 gene. Int J Mol Sci 2013, 14(9):18615-28.

23. Hao C, Wu B, Hou Z, Xie Q, Liao T, Wang T, Ma D: Asiatic acid inhibits LPS-induced inflammatory response in human gingival fibroblasts. International immunopharmacology 2017, 50:313-318.

24. Lian D, Dai L, Xie Z, Zhou X, Liu X, Zhang Y, Huang Y, Chen Y: Periodontal ligament fibroblasts migration injury via ROS/TXNIP/NIrp3 inflammasome pathway with Porphyromonas gingivalis lipopolysaccharide. Mol Immunol 2018, 103:209-219.

25. Darby IA, Hewitson TD: Fibroblast differentiation in wound healing and fibrosis. International review of cytology 2007, 257:143-179.

26. Croft AP, Campos J, Jansen K, Turner JD, Marshall J, Attar M, Savary L, Wehmeyer C, Naylor AJ, Kemble $\mathrm{S}$ et al: Distinct fibroblast subsets drive inflammation and damage in arthritis. Nature 2019, 570(7760):246-251.

27. Fukuda K, Ishida W, Fukushima A, Nishida T: Corneal Fibroblasts as Sentinel Cells and Local Immune Modulators in Infectious Keratitis. International journal of molecular sciences 2017, 18(9).

28. Baek KJ, Choi Y, Ji S: Gingival fibroblasts from periodontitis patients exhibit inflammatory characteristics in vitro. Archives of oral biology 2013, 58(10):1282-1292.

29. Scheres N, Laine ML, Sipos PM, Bosch-Tijhof CJ, Crielaard W, de Vries TJ, Everts V: Periodontal 
366

367

368

369

370

371

372

373

374

375

376

377

378

379

380

381

382

383

384

385

386

387

388

389

390

391

392 ligament and gingival fibroblasts from periodontitis patients are more active in interaction with Porphyromonas gingivalis. Journal of periodontal research 2011, 46(4):407-416.

30. Enzerink A, Vaheri A: Fibroblast activation in vascular inflammation. Journal of thrombosis and haemostasis : JTH 2011, 9(4):619-626.

31. Yi YS: Functional Role of Milk Fat Globule-Epidermal Growth Factor VIII in MacrophageMediated Inflammatory Responses and Inflammatory/Autoimmune Diseases. Mediators of inflammation 2016, 2016:5628486.

32. Green TD, Crews AL, Park J, Fang S, Adler KB: Regulation of mucin secretion and inflammation in asthma: a role for MARCKS protein? Biochimica et biophysica acta 2011, 1810(11):1110-1113.

33. Yang D, Postnikov YV, Li Y, Tewary P, de la Rosa G, Wei F, Klinman D, Gioannini T, Weiss JP, Furusawa $\mathrm{T}$ et al: High-mobility group nucleosome-binding protein 1 acts as an alarmin and is critical for lipopolysaccharide-induced immune responses. The Journal of experimental medicine 2012, 209(1):157-171.

34. Xie $\mathrm{P}$, Deng LX, Gong $\mathrm{P}$, Ding $\mathrm{Y}$, Tang $\mathrm{XH}$ : Expression of HMGB1 and HMGN2 in gingival tissues, GCF and PICF of periodontitis patients and peri-implantitis. Brazilian journal of microbiology : [publication of the Brazilian Society for Microbiology] 2011, 42(3):1213-1219.

35. Kim DY, Jun JH, Lee HL, Woo KM, Ryoo HM, Kim GS, Baek JH, Han SB: N-acetylcysteine prevents LPS-induced pro-inflammatory cytokines and MMP2 production in gingival fibroblasts. Archives of pharmacal research 2007, 30(10):1283-1292.

36. Gonciarz W, Krupa A, Hinc K, Obuchowski M, Moran AP, Gajewski A, Chmiela M: The effect of Helicobacter pylori infection and different $H$. pylori components on the proliferation and apoptosis of gastric epithelial cells and fibroblasts. PloS one 2019, 14(8):e0220636.

37. Wei F, Yang D, Tewary P, Li Y, Li S, Chen X, Howard OM, Bustin M, Oppenheim JJ: The Alarmin HMGN1 contributes to antitumor immunity and is a potent immunoadjuvant. Cancer research 2014, 74(21):5989-5998.

38. Yu J, Dong R, Da J, Li J, Yu F, Zha Y: High-Mobility Group Nucleosome-Binding Protein 1 Mediates Renal Fibrosis Correlating with Macrophages Accumulation and Epithelial-to- 
393

394

395

396

397

398

399

400

401

402

403

404

405

406

407

408

409

410

411

412

413

414

415

416

417

418

419

Mesenchymal Transition in Diabetic Nephropathy Mice Model. Kidney \& blood pressure research 2019, 44(3):331-343.

39. Arts RJW, Huang PK, Yang D, Joosten LAB, van der Meer JWM, Oppenheim JJ, Netea MG, Cheng SC: High-Mobility Group Nucleosome-Binding Protein 1 as Endogenous Ligand Induces Innate Immune Tolerance in a TLR4-Sirtuin-1 Dependent Manner in Human Blood Peripheral Mononuclear Cells. Front Immunol 2018, 9:526.

40. Li BZ, Zhang HY, Pan HF, Ye DQ: Identification of MFG-E8 as a novel therapeutic target for diseases. Expert opinion on therapeutic targets 2013, 17(11):1275-1285.

41. Nakaya M, Watari K, Tajima M, Nakaya T, Matsuda S, Ohara H, Nishihara H, Yamaguchi H, Hashimoto A, Nishida $\mathrm{M}$ et al: Cardiac myofibroblast engulfment of dead cells facilitates recovery after myocardial infarction. $J$ Clin Invest 2017, 127(1):383-401.

42. Li J, Xu X, Cai X, Weng Y, Wang Y, Shen Q, Shi X: Milk Fat Globule-Epidermal Growth FactorFactor 8 Reverses Lipopolysaccharide-Induced Microglial Oxidative Stress. 2019, 2019:2601394.

43. Aziz M, Jacob A, Matsuda A, Wu R, Zhou M, Dong W, Yang WL, Wang P: Pre-treatment of recombinant mouse MFG-E8 downregulates LPS-induced TNF- $\alpha$ production in macrophages via STAT3-mediated SOCS3 activation. PloS one 2011, 6(11):e27685.

Figure legends

Fig. 1 CM protected DF-1 chicken embryo fibroblasts from LPS-induced inflammation and cell injury. (A-C) DF-1 chicken embryo fibroblasts were treated with different concentrations of LPS for $6 \mathrm{~h}$. Next, TNF- $\alpha$ and IL-6 secretion levels were examined by corresponding ELISA kits. cell viability was detected by CCK-8 assay. (D and E) DF-1 chicken embryo fibroblasts were pre-treated with $10 \mu \mathrm{g} / \mathrm{ml}$ of LPS for $6 \mathrm{~h}$ and then stimulated with different doses of CM for $24 \mathrm{~h}$. TNF- $\alpha$ and IL-6 secretion levels were detected by corresponding ELISA kits. (F) DF-1 chicken embryo fibroblasts were pre-treated with $10 \mu \mathrm{g} / \mathrm{ml}$ of LPS for $6 \mathrm{~h}$ and then stimulated with different doses of $\mathrm{CM}$. At $0,24,48,72 \mathrm{~h}$ after $\mathrm{CM}$ exposure, cell viability was detected by CCK-8 assay. Untreated cells functioned as the control group. (A-E): one-way ANOVA (Tukey's post-hoc test). F: two-way ANOVA (Sidak post hoc test). Each group sample contained 3 replicates.

PeerJ reviewing PDF | (2021:01:57494:1:1:NEW 2 Jul 2021) 
420

421

422

423

424

425

426

427

428

429

430

431

432

433

434

435

436

437

Fig. 2 Identification of dysregulated proteins in response to LPS stimulation alone or in combination with CM in DF-1 chicken embryo fibroblasts. (A) Venn analysis for down-regulated proteins in DL vs D group and up-regulated proteins in DLE vs DL group. (B) Venn analysis for up-regulated proteins in DL vs D group and down-regulated proteins in DLE vs DL group. (C-E) The top 20 terms enriched by proteins with the converse change trends in DLE vs DL and DL vs D groups based on biological processes, cellular components, and molecular functions. (F) The top 20 KEGG pathways enriched by proteins with converse change trends in DLE vs DL and DL vs D groups. DL: DF-1 cell group treated with LPS alone; D group: DF-1 cell group without any treatment; DLE group: DF-1 cell group co-treated with LPS and CM.

Fig. 3 Effects of LPS alone or in combination with CM on the expression of MFGE8, MARCKS, HMGN1, and HMGN2 in DF-1 chicken embryo fibroblasts. (A-D) DF-1 chicken embryo fibroblasts were stimulated with LPS $(10 \mu \mathrm{g} / \mathrm{ml})$ for $6 \mathrm{~h}$ and then treated with CM $(20 \mu \mathrm{M})$ for $24 \mathrm{~h}$. Next, mRNA expression levels of MFGE8, MARCKS, HMGN1, and HMGN2 were measured by RT-qPCR assay. ${ }^{*} \mathrm{P}<0.05 . * * \mathrm{P}<0.01$. (A-D): one-way ANOVA (Tukey’s post-hoc test).

Fig. 4 CM exerted its anti-inflammatory and pro-survival activities by regulating MFGE8 and HMGN1 expression in LPS-treated DF-1 chicken embryo fibroblasts. (A and B) DF-1 chicken embryo fibroblasts were transfected with si-NC, si-MFGE8\#1, si-MFGE8\#2, si-HMGN1\#1 or si-HMGN1\#2. MFGE8 and HMGN1 mRNA levels were determined by RT-qPCR assay at $48 \mathrm{~h}$ post transfection. (C and) Transfected DF1 chicken embryo fibroblasts were treated with LPS (10 $\mu \mathrm{g} / \mathrm{ml})$ for $6 \mathrm{~h}$ and CM (20 $\mu \mathrm{M})$ for $24 \mathrm{~h}$. TNF- $\alpha$ and IL-6 secretions were measured by corresponding ELISA kits. (E) DF-1 chicken embryo fibroblasts transfected with si-NC, si-MFGE8\#1 or si-HMGN1\#2 were treated with LPS (10 $\mu \mathrm{g} / \mathrm{ml})$ for $6 \mathrm{~h}$ and stimulated with CM $(20 \mu \mathrm{M})$ for $0,24,48$, or $72 \mathrm{~h}$. Cell viability was examined via CCK-8 assay. (A-D): one-way ANOVA (Tukey’s post-hoc test). E: two-way ANOVA (Sidak post hoc test).

Supplementary Fig. 1 Heat map of the proteins with converse change trends in DLE vs DL and DL vs D groups.

Supplementary Fig. 2 The potential link between TMPRSS2 and MFGE8 (or HMGN1) was speculated using the STITCH website (http://stitch.embl.de/cgi/). 
447

448 Supplementary Table 1 The sense sequences of siRNAs

449 Supplementary Table 2 The information of proteins corresponding to the heat map of Supplementary Fig. 1.

450 Supplementary Table 3 GO enrichment analysis for proteins with converse change trends in DLE vs DL and

451 DL vs D groups based on biological processes, cellular components, and molecular functions.

452 Supplementary Table 4 KEGG enrichment analysis for proteins with converse change trends in DLE vs DL 453 and DL vs D groups.

454 Supplementary Table 5 Annotation analysis for MFGE8, MARCKS, HMGN1, HMGN2 by KOBAS and 455 Uniport databases.

456

457 
Figure 1

Revised_Figure_1

Fig. 1 CM protected DF-1 chicken embryo fibroblasts from LPS-induced inflammation and cell injury.

A

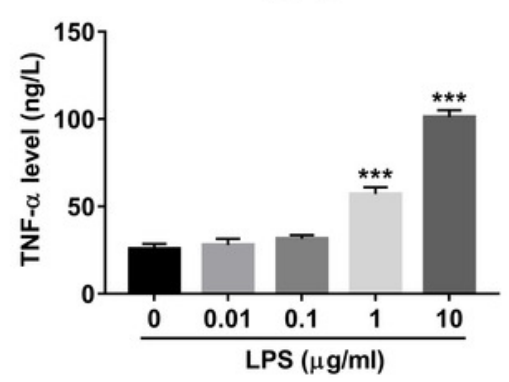

D
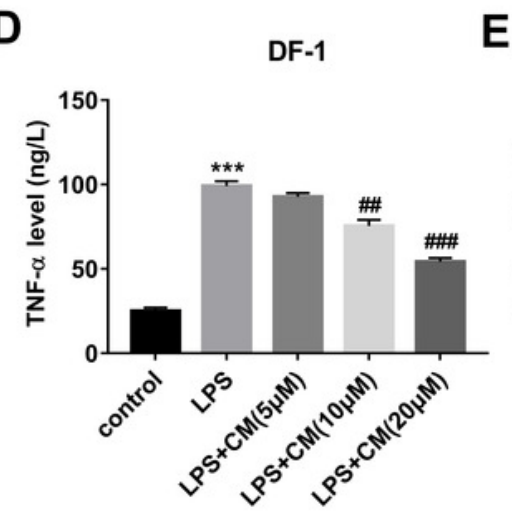

B

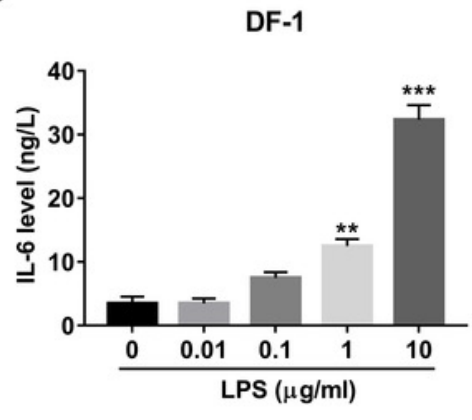

E

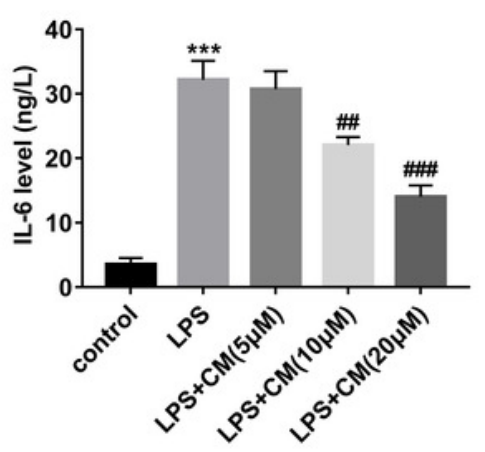

C

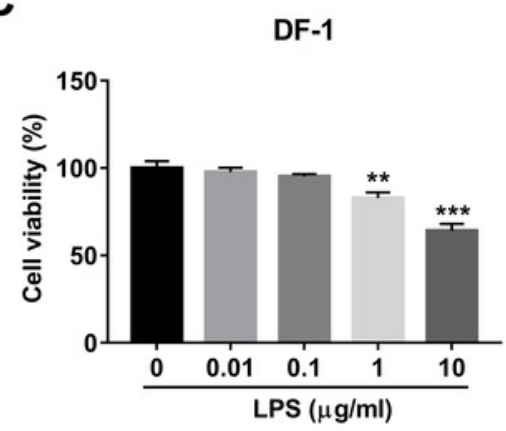

F

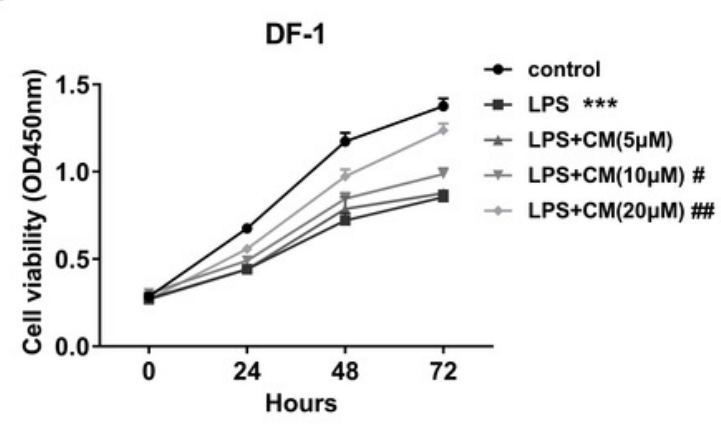


Figure 2

Revised_Figure_2

Fig. 2 Identification of dysregulated proteins in response to LPS stimulation alone or in combination with CM in DF-1 chicken embryo fibroblasts. 
A

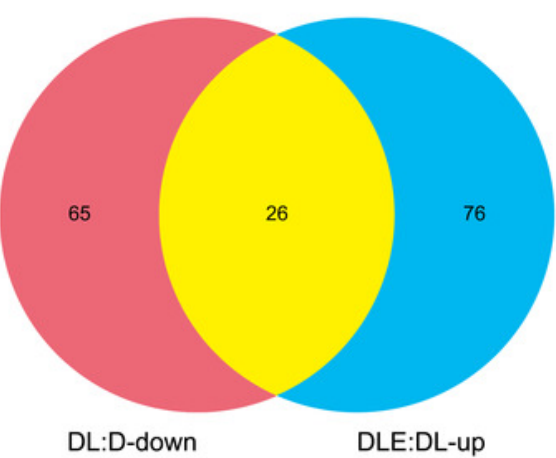

C

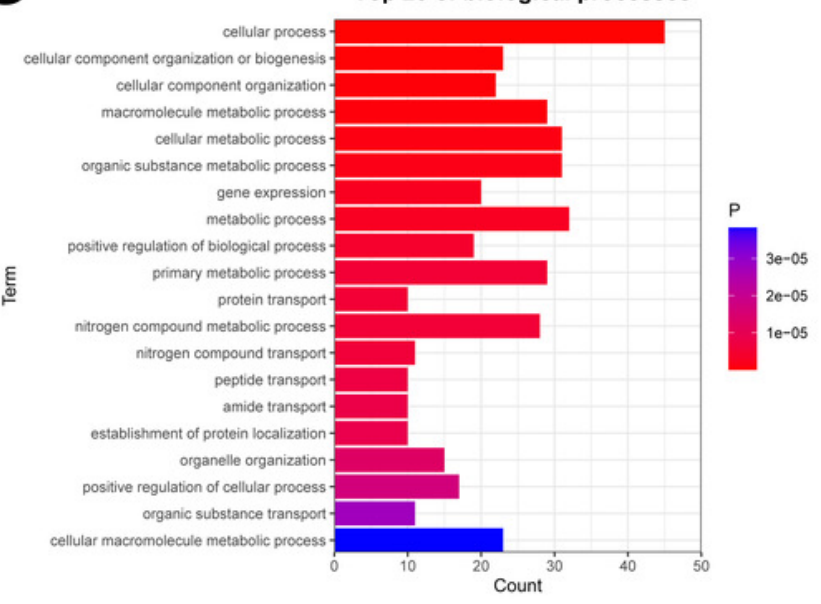

E

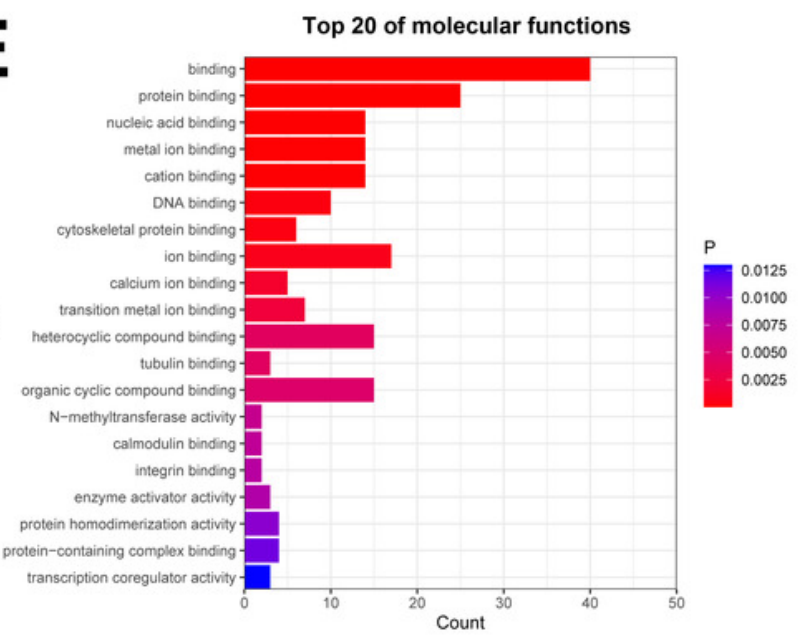

B
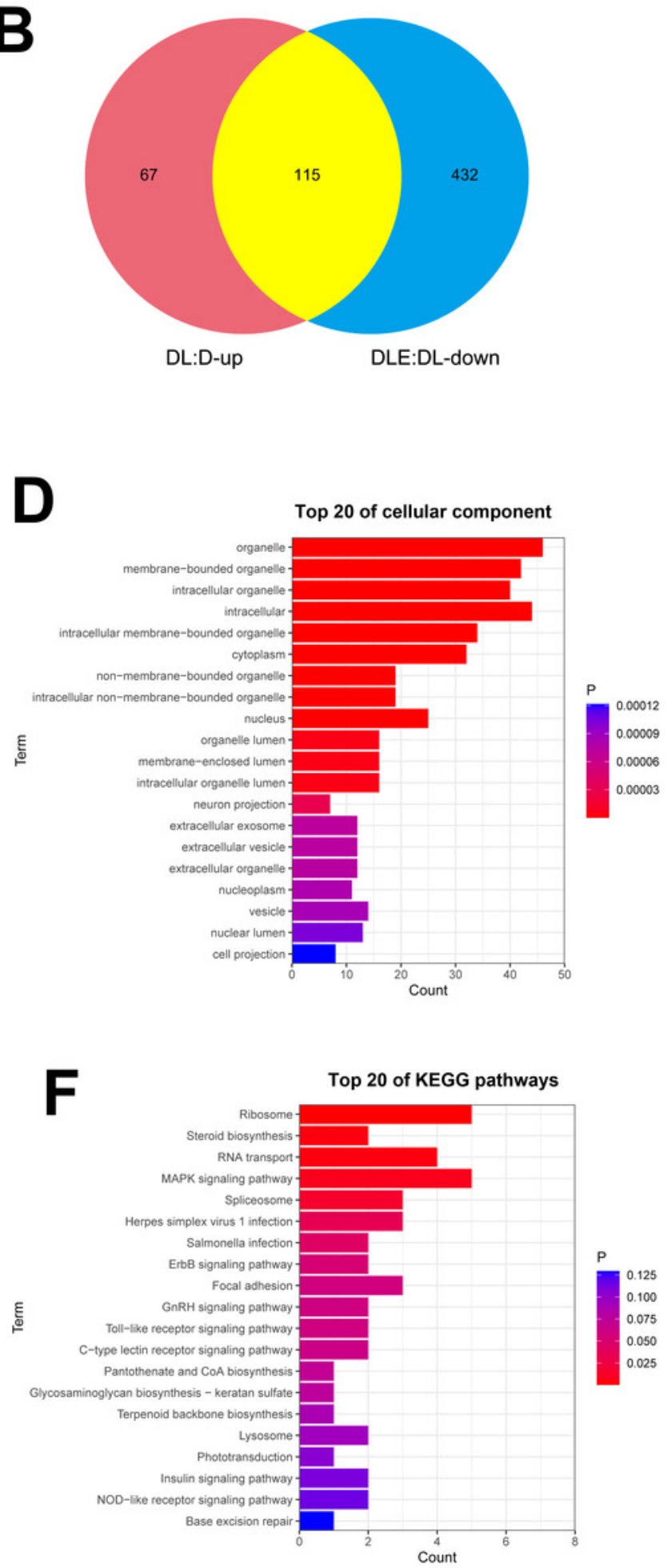
Figure 3

Figure_3

Fig. 3 Effects of LPS alone or in combination with CM on the expression of MFGE8, MARCKS, HMGN1, and HMGN2 in DF-1 chicken embryo fibroblasts.

A

40

0.

C

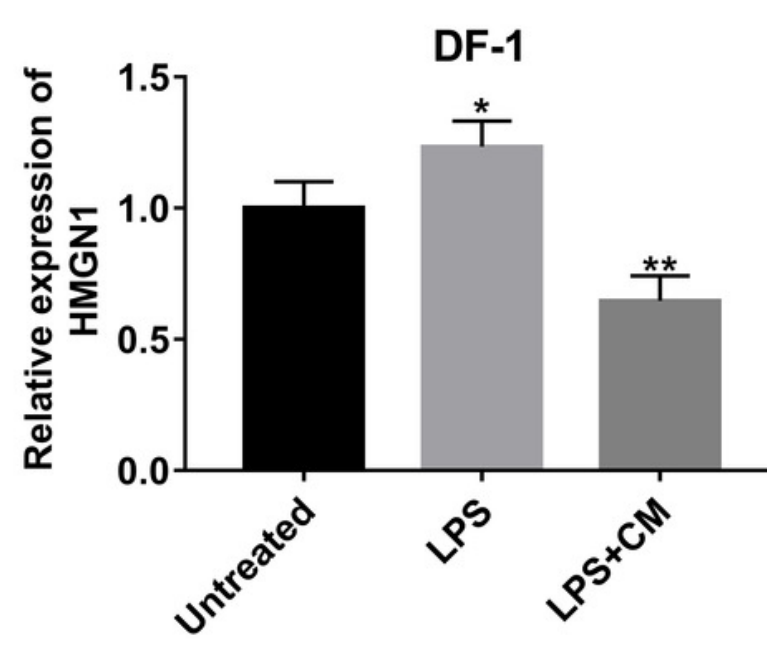

DF-1

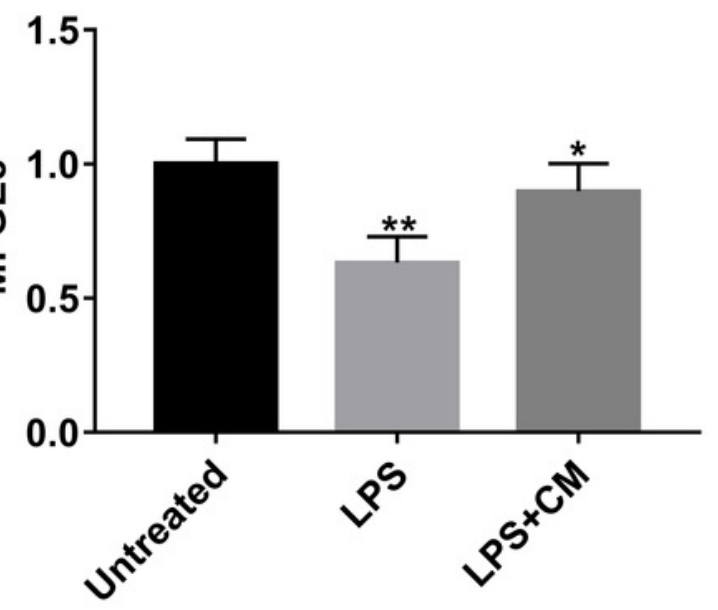

D
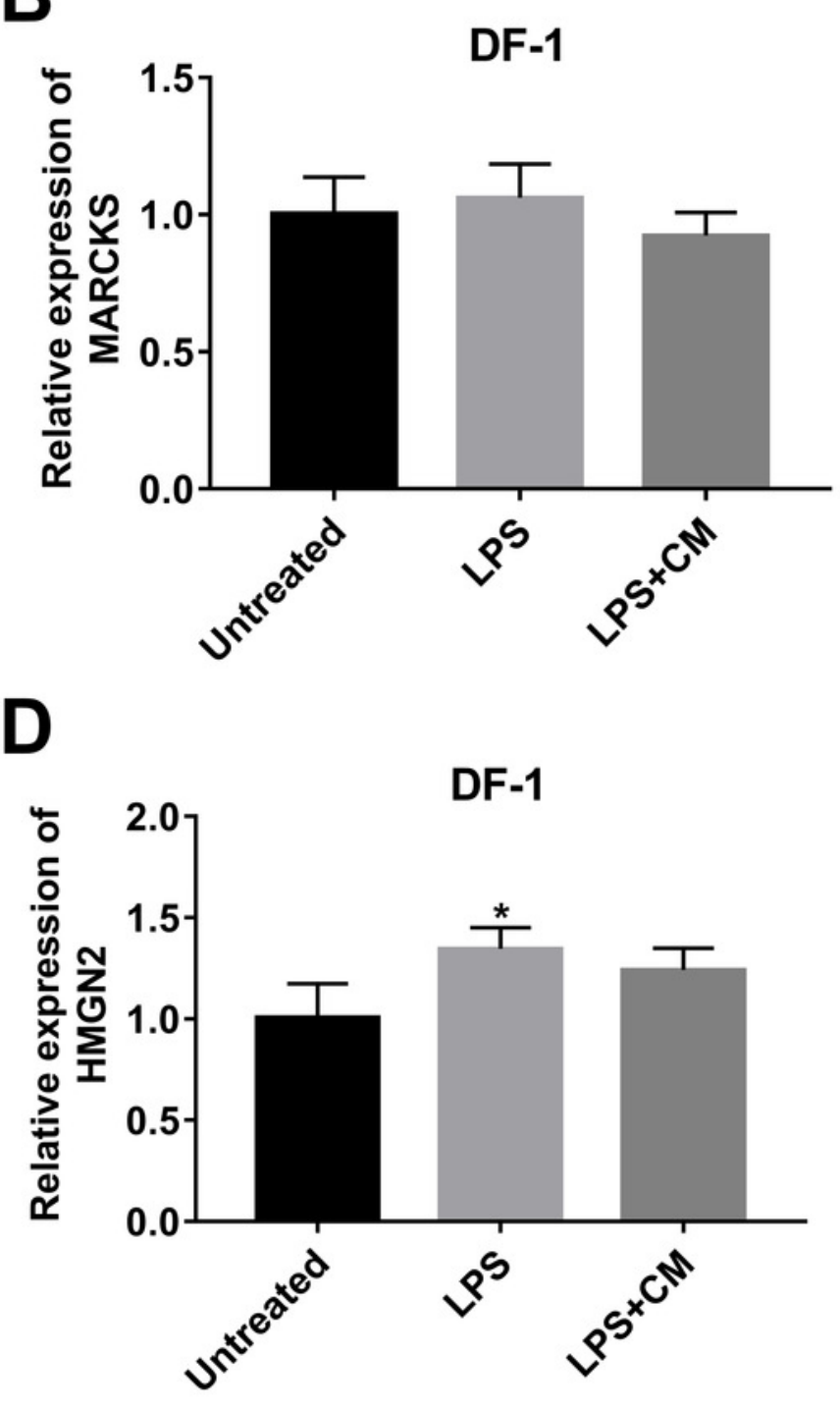
Figure 4

Revised_Figure_4

Fig. 4 CM exerted its anti-inflammatory and pro-survival activities by regulating MFGE8 and HMGN1 expression in LPS-treated DF-1 chicken embryo fibroblasts.

A

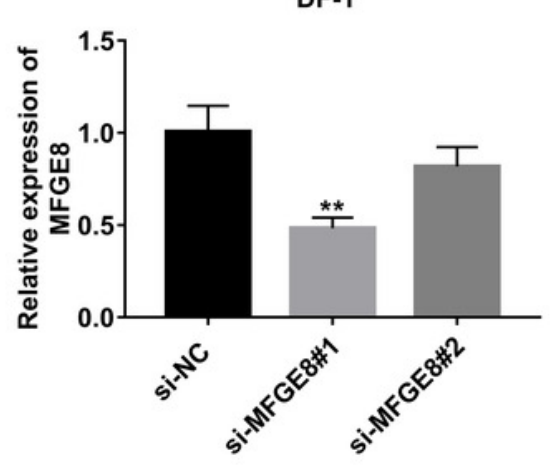

D

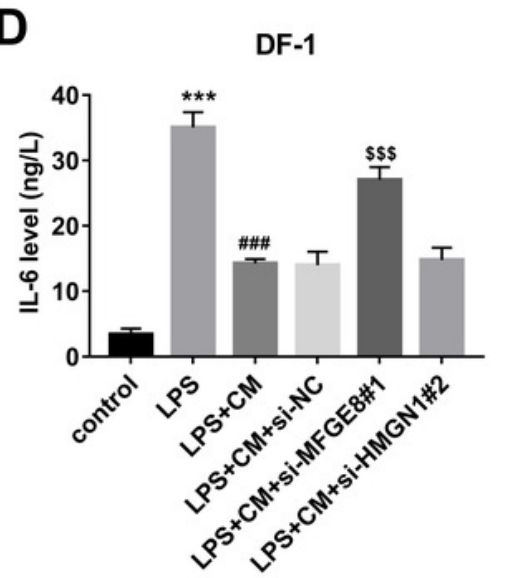

B

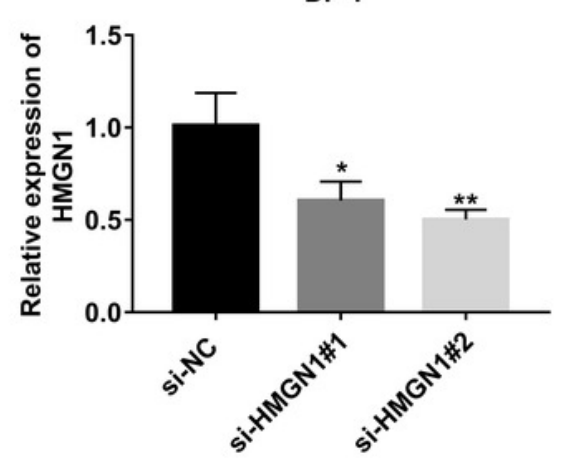

E

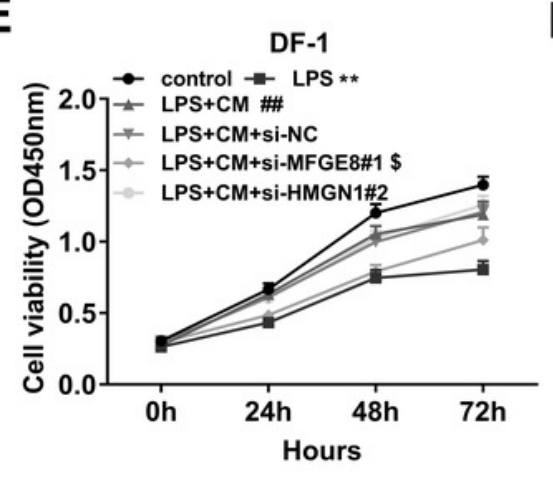

C

$\mathbf{F}$ DF-1
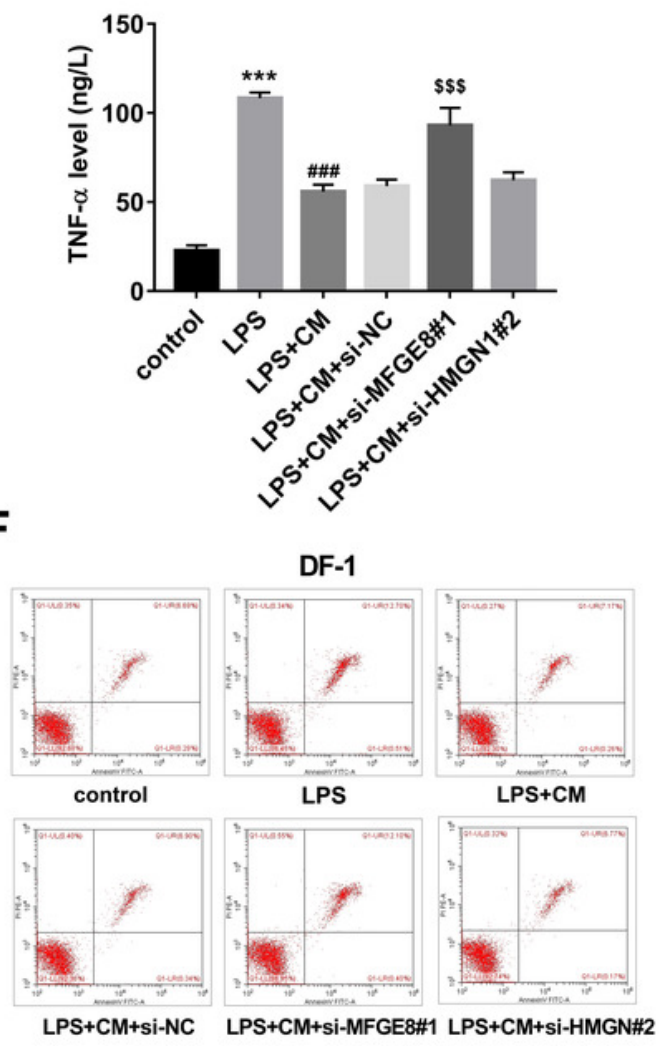